\title{
An aberrant anterior lobe and unusual accessory fissure of the left lung in human - anatomical and clinical considerations
}

\author{
Lazar Jelev, Dimka Hinova-Palova, Wladimir Ovtscharoff \\ Department of Anatomy, Histology and Embryology, Medical University of Sofia, Sofia, Bulgaria
}

\begin{abstract}
Accessory fissures that separate aberrant lung lobes are not only interesting anatomical observations, but also have a definite importance as they may cause diagnostic confusion in radiological and pathological evaluations. In the case reported here, a rare type of separation of the left lung by an unusual fissure is described. The left lung of a 67-year-old Caucasian female cadaver showed an accessory fissure that crossed the mediastinal, apical and anterior costal surfaces. The aberrant fissure separated the organ into a small upper-medial-anterior lung lobe (lobus minimus) and a much larger aberrant lobe (lobus magnus). At the pulmonary hilum, the artery, vein and bronchus of the small aberrant lobe were clearly identifiable. During analysis of radiological images such as X-ray and computed tomography, the existence of such aberrant fissures and lobes have to be known in detail in order to separate between simple anatomic variation of the lungs and a number of lung pathologies including scar, pulmonary bulla, atelectasis or even mediastinal mass.
\end{abstract}

Keywords: aberrant lung lobe; accessory lung fissure; human; variation

Anatomy 2015;9(3):192-195 @2015 Turkish Society of Anatomy and Clinical Anatomy (TSACA)

\section{Introduction}

The smooth and shiny outer surface of the lungs is typically interrupted by deep clefts called interlobar fissures that separate the major lung lobes. ${ }^{[1]}$ Commonly, there are two lobes of the left lung which are separated by the oblique fissure (left major fissure) and three lobes of the right lung separated by the oblique (right major) and transverse (minor) fissures. ${ }^{[1]}$ During routine anatomical dissections of human cadavers, different pathologies can be observed including tight adhesions between the interlobar surfaces that may hamper identification of the lung fissures and lobes. In some rare cases, there might be an aberrant congenital lung division with the presence of incomplete, missing or even additional lung fissures. ${ }^{[2]} \mathrm{In}$ clinical practice, such accessory or aberrant lung fissures may be misinterpreted as lung pathologies on $\mathrm{X}$-rays and CT scans of the chest. ${ }^{[2]}$ Herewith, we present an interesting case of aberrant lung fissure separating the left lung into two unusual lobes, with an emphasis on the anatomical basis of this anomaly and its possible significance in clinical practice.

\section{Case Report}

After completion of the routine anatomical dissection of the thoracic wall of a 67-year-old Caucasian female cadaver, the heart and lungs were removed. The removal of the left lung was complicated due to pleural adhesions on the dorsal aspect of its costal surface that caused some tearing of the lung parenchyma. Careful examination of the left lung revealed the presence of an accessory fissure that separated the organ into two aberrant lobes in quite different sizes. The aberrant fissure was well seen on the apical, anterior costal and mediastinal surfaces of the lung (Figures 1a-c). This unusual fissure started above the pulmonary hilum on the mediastinal surface and ascended to the apex splitting it into an anteromedial and a posterolateral part. Then, the fissure descended vertically along the anterior costal surface, parallel to the 

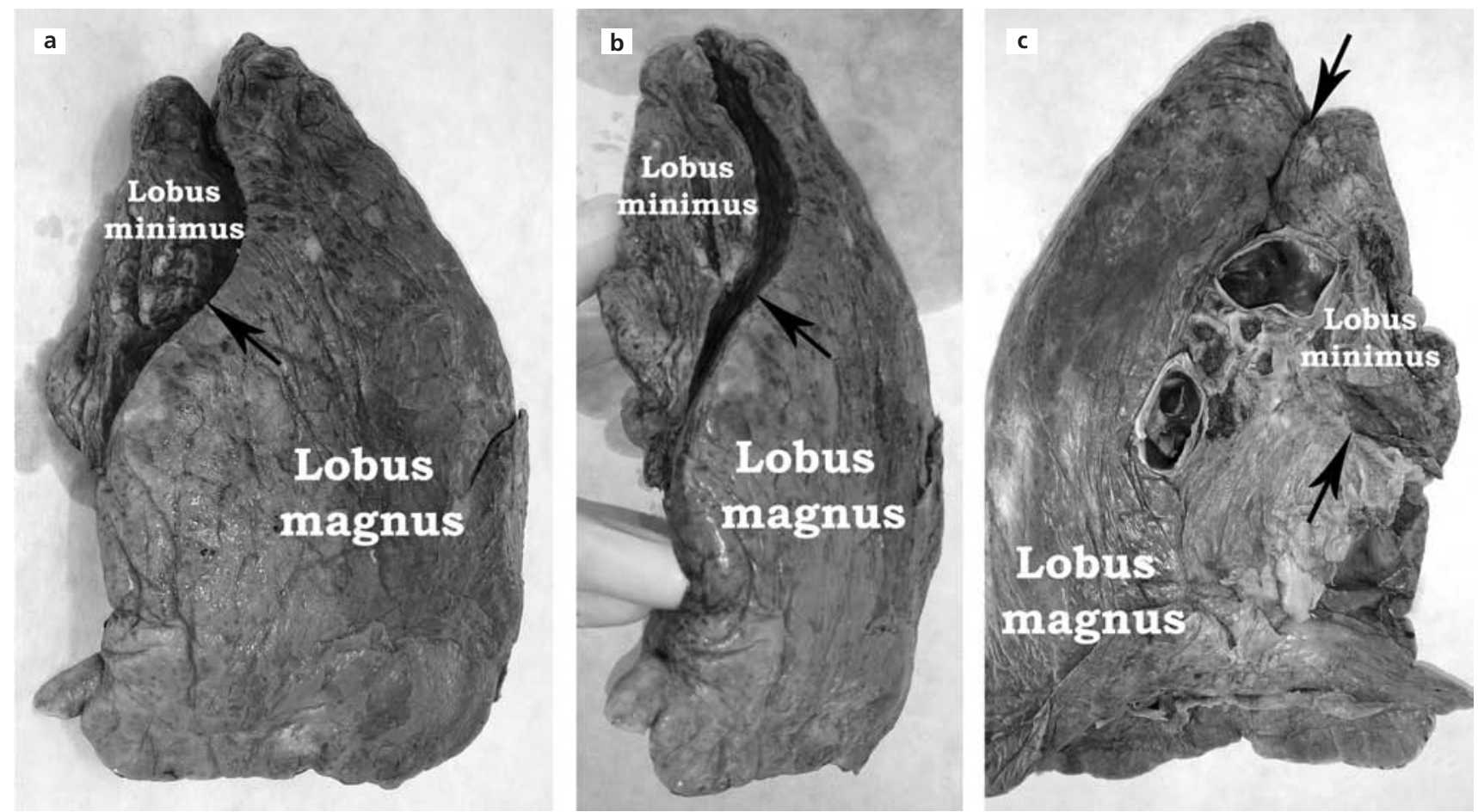

Figure 1. Lateral (a) and anterior (b) view of the costal surface and mediastinal surface (c) of the left lung with the accessory fissure (arrows).

anterior border in a slightly curved direction with a lateral convexity. Nearing the midpoint of the anterior border, the aberrant fissure turned back and reached the anterior part of the pulmonary hilum. Thus, the aberrant fissure separated a small upper-medial-anterior lung lobe (lobus minimus) from a much larger aberrant lobe (lobus magnus). The latter was probably composed of the fused lower-posterior part of superior lobe and the inferior lobe. A short secondary fissure split the anterior border of the small aberrant lobe. The cardiac notch belonged to the large aberrant lobe with the lingula clearly visible. The usual oblique fissure was not observed in detailed examination of the costal surface as well as the dorsal aspect of the left lung. At the left pulmonary hilum, the main structures to the aberrant lobes (lobus minimus and magnus) were clearly identifiable. Well separated pulmonary artery, vein and bronchus to the small upper-medial-anterior lobe were observed (Figure 2). Also, examining the surface of the heart atria, a well separated small orifice for the pulmonary vein of the aberrant small left lobe was identified (Figure 3). On the right side, there were also pleural adhesions around the lung, but after its removal from the chest, three lobes separated by the usual two fissures were observed.

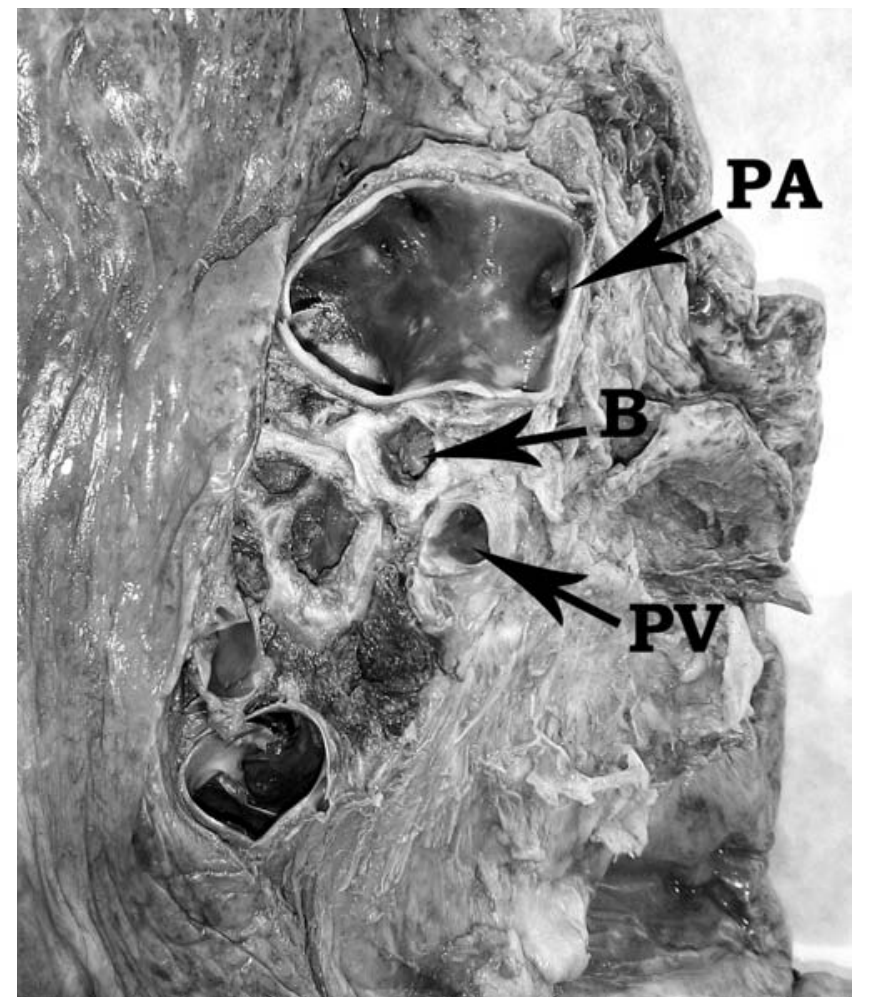

Figure 2. Closer view of the mediastinal surface and the hilum area of the left lung with arrows showing the separate pulmonary artery (PA), pulmonary vein (PV) and lobar bronchus (B) to the small aberrant lung lobe. 


\section{Discussion}

Anatomically, an "accessory" lung lobe is a part of the lung that is separated by an "accessory" fissure. ${ }^{[2]}$ Despite its name, the accessory lung lobe does not provide an extra tissue to the lung; it is a result of an unusual congenital separation of the primary bronchial bud. ${ }^{[3]}$ The accessory lung fissures can be found in up to $50 \%$ of lung specimens, but are less frequently demonstrated on radiographs and CT scans. ${ }^{[2,4]}$ The most common accessory fissures of the lung are the inferior accessory fissure which separates the medial basal segment (S VII), the superior accessory fissure which separates the superior segment (S VI), the left minor fissure which separates the lingula (S IV and S V), and the azygos fissure that demarcates the azygos lobe of the right upper lobe. ${ }^{[2,5,6]} \mathrm{A}$ number of other rare aberrant fissures are also described in the literature. ${ }^{[2,7,8]}$ The accessory lung fissures usually exist between the bronchopulmonary segments that are otherwise normal. ${ }^{[2]}$ The azygos lobe in the right upper lobe, however, does not correspond to a cleft between segments; it is a result of abnormal disposition of the azygos vein during embryonic development. ${ }^{[5]}$

Considering the normal segmental anatomy of the left lung and the territories of the usual bronchopulmonary segments, ${ }^{[9]}$ the small aberrant upper-medialanterior lobe (lobus minimus) described in our case is probably composed of the apical segment (S I) and the ventral part of the anterior segment (S III). The large aberrant lung lobe (lobus magnus) is composed of the segments S II, dorsal part of S III and S IV - S X. The incidence of this accessory fissure and corresponding lobes is difficult to estimate. We have observed only one case in over 100 dissected bodies, suggesting the incidence is less than $1 \%$. In their detailed study, Berkmen et al. ${ }^{[7]}$ also found accessory fissures of the left upper lobe very rare.

The accessory lung fissure appears as a thin white line on the chest radiography resembling the normal lung fissures except for their unusual location. ${ }^{[2]}$ On CT scans in different planes, accessory fissures and accessory lobes may be examined in detail. ${ }^{[2,7,8]}$ Sometimes, however, the radiographic image of an aberrant fissure can be mistaken for a scar, the wall of a pulmonary bulla or a pleural line made visible by pneumothorax. ${ }^{[2]}$ The accessory fissure may serve as a barrier to the spreading infection. In such cases of pneumonia of the accessory lobe, the well separated lobe may be confused with atelectasis, consolidation of the lung parenchyma or even mediastinal mass. $^{[2,8]}$

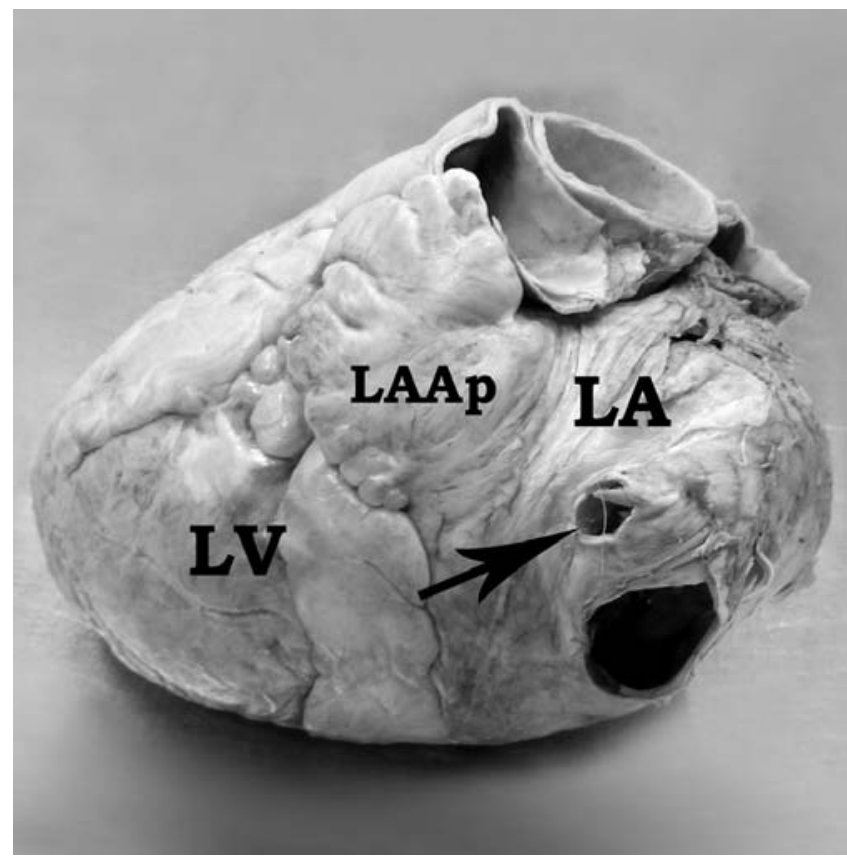

Figure 3. Left lateral view of the heart with clearly visible separate orifice (arrow) of the pulmonary vein of the small aberrant lung lobe. LA: left atrium; LAAp: left atrial appendage; LV: left ventricle.

\section{Conclusion}

It is important to recognize the presence of accessory lung fissures and lobes to detect them on radiographs or CT scans. This is in order to separate them from other lung pathologies including such as scar, pulmonary bulla, atelectasis or even mediastinal mass. This report presents a rare case of an aberrant anterior lobe and unusual accessory fissure of the left lung.

\section{References}

1. Clemente CD, editor. Anatomy of the human body. 30th ed. Philadelphia (PA): Lea and Febiger; 1985. p. 1385-401.

2. Godwin JD, Tarver RD. Accessory fissures of the lung. AJR Am J Roentgenol 1985;144:39-47.

3. Foster-Carter AF. Broncho-pulmonary abnormalities. Br J Tuberc Dis Chest 1946;40:111-24.

4. Ariyurek OM, Gulsun M, Demirkazik FB. Accessory fissures of the lung: evaluation by high-resolution computed tomography. Eur Radiol 2001;11:2449-53.

5. Mata J, Cáceres J, Alegret X, Coscojuela P, De Marcos JA. Imaging of the azygos lobe: normal anatomy and variations. AJR Am J Roentgenol 1991;156:931-7.

6. Shields TW. Surgical anatomy of the lungs. In: Shields TW, editor. General Thoracic Surgery. 5th ed. Philadelphia: Lippincott Williams and Wilkins; 2000. p. 63-75.

7. Berkmen T, Berkmen YM, Austin JH. Accessory fissures of the upper lobe of the left lung: CT and plain film appearance. AJR Am J Roentgenol 1994;162:1287-93. 
8. Cronin P, Gross BH, Kelly AM, Patel S, Kazerooni EA, Carlos RC Normal and accessory fissures of the lung: evaluation with contiguous volumetric thin-section multidetector CT. Eur J Radiol 2010;75: e1-8.
9. Schünke M, Schulte E, Schumacher U, editors. Prometheus. Lernatlas der Anatomie. Hals und Innere Organe. Stuttgart: Georg Theime Verlag; 2005. p. 84-5.

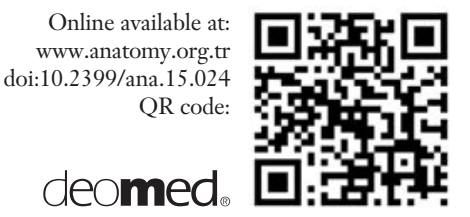

Correspondence to: Lazar Jelev, MD, PhD

Department of Anatomy, Histology and Embryology, Medical University

of Sofia, blvd. Sv. Georgi Sofiisky 1, BG-1431 Sofia, Bulgaria

Phone: +9035929172636

e-mail: ljelev@abv.bg

Conflict of interest statement: No conflicts declared.

This is an open access article distributed under the terms of the Creative Commons Attribution-NonCommercial-NoDerivs 3.0 Unported (CC BY-NCND3.0) Licence (http://creativecommons.org/licenses/by-nc-nd/3.0/) which permits unrestricted noncommercial use, distribution, and reproduction in any medium, provided the original work is properly cited. Please cite this article as: Jelev L, Hinova-Palova D, Ovtscharoff W. An aberrant anterior lobe and unusual accessory fissure of the left lung in human - anatomical and clinical considerations. Anatomy 2015;9(3):192-195. 